\title{
Toxicidade diferencial de agrotóxicos utilizados em citros para Neoseiulus californicus, Euseius concordis e Brevipalpus phoenicis
}

\author{
Marcos Zatti da Silva $\left({ }^{1 *}\right)$; Mário Eidi Sato $\left({ }^{2}\right)$; Carlos Amadeu Leite de Oliveira ( $)$; Débora Soller Rais $\left({ }^{2}\right)$ \\ (') Departamento de Fitossanidade, Faculdade de Ciências Agrárias e Veterinárias - FCAV/UNESP, Via de acesso Professor Paulo \\ Donato Castellane km 5, 14884-900 Jaboticabal (SP) E-mail: makdsil@ig.com.br (*) Autor correspondente; Bolsista de doutorado \\ CAPES; amadeu@unesp.com.br
}

(2) Instituto Biológico, APTA, Rodovia Heitor Penteado, km 3, 13092-543 Campinas (SP) E-mail: mesato@biologico.sp.gov.br; debysrais@gmail.com

Recebido: 8/jun./2009; Aceito: 1/jun./2010

\begin{abstract}
Resumo
Brevipalpus phoenicis (Geijskes) (Acari: Tenuipalpidae) é considerada uma das principais pragas em citros no Brasil, devido à transmissão do vírus da leprose dos citros e à necessidade de frequentes aplicações de acaricidas para seu controle. Assim, objetivou-se comparar a toxicidade dos principais agrotóxicos utilizados em citros no Brasil, sobre populações de B. phoenicis e de ácaros predadores das espécies Neoseiulus californicus (McGregor) e Euseius concordis (Chant) (Acari: Phytoseiidae). Foram avaliadas duas populações de E. concordis: uma procedente de Jaboticabal (SP) e outra de Descalvado (SP). Arenas de folhas (para fitoseídeos) ou frutos (para B. phoenicis) de Citrus sinensis Osbeck (L.) CV. Pera, contendo 25 fêmeas adultas de cada espécie foram pulverizadas em torre de Potter. As avaliações de mortalidade foram realizadas 72 horas após a aplicação. Foram utilizadas 4 a 6 concentrações de cada produto (abamectina, acefato, azociclotina, bifentrina, clorfenapir, dicofol, dinocape, óxido de fembutatina, propargito e piridabem) para a obtenção das curvas de concentração-resposta. Abamectina, azociclotina, clorfenapir, dicofol, dinocape, óxido de fembutatin, propargito e piridabem foram altamente tóxicos a B. phoenicis, com valores de $\mathrm{CL}_{90}$ iguais ou inferiores aos das concentrações recomendadas desses produtos. $N$. californicus foi mais tolerante a bifentrina, propargito, óxido de fembutatina e dinocape que B. phoenicis e E. concordis (ambas as populações). Esses acaricidas tiveram baixa toxicidade aos adultos de $\mathrm{N}$. californicus, para o qual as $\mathrm{CL}_{50}$ foram pelo menos seis vezes maiores que suas concentrações recomendadas. Acefato foi extremamente tóxico a E. concordis, mas inócuo a adultos de N. californicus. A população de E. concordis de Jaboticabal foi mais resistente que a de Descalvado a azociclotina, bifentrina, clorfenapir, dicofol e propargito.
\end{abstract}

Palavras-chave: ácaro predador, ácaro da leprose, fitoseídeo, acaricidas, resistência.

\section{Differential toxicity of pesticides used on citrus to Neoseiulus californicus, Euseius concordis and Brevipalpus phoenicis}

\begin{abstract}
Brevipalpus phoenicis (Geijskes) is considered one of the most important pests on citrus in Brazil, due to the transmission of Citrus leprosis virus and the necessity of frequent acaricide applications for its control. This study was carried out to compare the toxicity of the main pesticides used on citrus in Brazil to the populations of B. phoenicis and the predaceous mites Neoseiulus californicus (McGregor), Euseius concordis (Chant). Two populations of E. concordis were evaluated: one from Jaboticabal County and other from Descalvado County, State of São Paulo. Arenas of leaves (for phytoseiids) and fruits (for B. phoenicis) of Citrus sinensis (L.) Osbeck cv. Pera with 25 adult females of each species were sprayed under Potter spray tower. The mortality was assessed 72 hours after treatment. Four to six concentrations of each chemical (abamectin, acephate, azocyclotin, bifenthrin, chlorfenapyr, dicofol, dinocap, fenbutatin oxide, propargite and pyridaben) were used to obtain the concentration-mortality curves. Abamectin, azocyclotin, chlorfenapyr, dicofol, dinocap, fenbutatin oxide, propargite and pyridaben were highly toxic to $B$. phoenicis, presenting $L C_{90}$ values equal or lower than the recommended concentrations of the chemicals. $N$. californicus was more tolerant to bifenthin, propargite, fenbutatin oxide and dinocap than B. phoenicis and E. concordis (both populations). These pesticides presented low toxicity to the adults of $\mathrm{N}$. californicus, for which the $\mathrm{LC}_{50}$ of chemicals were at least six times higher than their recommended concentrations. Acephate was extremely toxic to E. concordis from Descalvado and Jaboticabal, but innocuous to adults of $\mathrm{N}$. californicus. The population of E. concordis from Jaboticabal was more resistant to azocyclotin, bifenthrin, chlorfenapyr, dicofol and propargite than that from Descalvado.
\end{abstract}

Key words: predatory mite, leprosis mite, phytoseiid, acaricides, resistance. 


\section{INTRODUÇÃO}

Brevipalpus phoenicis (Geijskes) é um ácaro-praga responsável por uma parcela significativa do custo da produção de citros no Brasil, devido à necessidade de frequentes aplicaçôes de acaricidas para seu controle (Neves et al., 2004). Um dos problemas causados por essas frequentes aplicaçóes é o desenvolvimento da resistência de ácaros-praga (Омото et al., 2000). Outro problema associado ao uso excessivo de agrotóxicos é a eliminação dos inimigos naturais, tendo como conseqüência a ressurgência de pragas e intenso desequilíbrio ecológico (vAN DE VRIE et al., 1972).

Um dos possíveis problemas relacionados à liberação de ácaros predadores visando ao controle de ácaros-praga seria o fato de que estes inimigos naturais poderiam ser mortos devido à aplicação de agrotóxicos. Neste caso, o emprego de populaçôes de fitoseídeos tolerantes ou resistentes a produtos químicos poderia ser interessante em programas de manejo de ácaros em diversas culturas (Hoy, 1985).

A resistência entre os artrópodes fitófagos e seus inimigos naturais proporciona efeitos contrastantes, e para os fitófagos a resistência intensifica sua condição de praga reduzindo as possibilidades de manejo. Por outro lado, a evoluçấo da resistência em populaçôes de inimigos naturais pode contribuir de maneira significativa para o manejo integrado de pragas (MIP) pela conservação desses organismos mesmo após aplicações de produtos considerados nocivos a eles (CROFT, 1990).

Embora a resistência a agrotóxicos não seja frequente em inimigos naturais, atualmente são conhecidos diversos casos de resistência em ácaros predadores da família Phytoseiidae como em Amblyseius fallacis (Garman), Euseius concordis (Chant), Iphiseiodes zuluagai Denmark \& Muma, Metaseiulus occidentalis (Nesbitt), Phytoseiulus persimilis Athias-Henriot, Typhlodromus pyri Scheuten (STRICKLER E CrofT, I982; Hoy, I985; Marwick, I986; Poletti, 2002).

Uma população de ácaros fitoseídeos da espécie Neoseiulus californicus (McGregor), coletada em morangueiro comercial em Atibaia (SP), foi tolerante ou resistente a diversos agrotóxicos utilizados em citros no Brasil (SiLVA e Oliveira, 2006; 2007). Essa espécie de fitoseídeo é fácil de ser criada e é eficiente na predação de ovos (SiLVA, 2005) e larvas de $B$. phoenicis. A utilização de ácaros dessa população de $N$. californicus em programas de manejo de ácaros pode ser bastante interessante em pomares de citros do Estado de São Paulo, onde o uso de agrotóxicos é bastante intenso.

A introdução de fitoseídeos cuja resistência foi desenvolvida em campo, para novas regióes geográficas, tem frequentemente resultado em um controle biológico efetivo (Penman et al., 1979), sendo uma forma análoga ao controle biológico clássico, porém utilizando um biótipo diferenciado de inimigo natural (Dunley et al., 1991).

Desta forma, objetivou-se comparar a toxicidade dos principais agrotóxicos utilizados na cultura de citros, no Brasil, sobre populações de $N$. californicus, E. concordis e B. phoenicis, para se obter subsídios para o manejo de ácaros pragas em citros.

\section{MATERIAL E MÉTODOS}

\section{Origem e criação dos ácaros utilizados nos bioensaios}

Brevipalpus phoenicis - A população original de $B$. phoenicis, coletada de plantas de citros, no município de Piracicaba (SP), foi cedida pelo Prof. Dr. Celso Omoto. Estes ácaros foram criados sobre frutos de laranja doce [Citrus sinensis (L.) Osbeck] cv. Pera, coletados em pomares livres da aplicação de acaricidas. Os frutos foram lavados com água e, após a secagem, parafinados, deixando-se uma arena de $6 \mathrm{~cm}^{2}$ circundada com cola adesiva (Tanglefoot ${ }^{\circ}$ ) para conter os ácaros. Em cada fruto, foram transferidos de 40 a 50 ácaros adultos com auxílio de um pincel de pelo macio. Os frutos foram substituídos a cada 30 a 40 dias, por outros mais novos, com as mesmas características.

Neoseiulus californicus - A população de N. californicus utilizada neste trabalho foi coletada em cultivo comercial de morangueiro, no município de Atibaia (SP), em 30/10/1999.

Manteve-se uma criação-estoque de $N$. californicus no laboratório sobre plantas de feijão-de-porco [Canavalia ensiformis (L.)] cultivadas em vasos plásticos de $500 \mathrm{~mL}$, tendo como fonte alimentar Tetranychus urticae Koch. A criação foi, posteriormente, transferida para discos de folha de laranjeira da variedade Pera, com aproximadamente $7 \mathrm{~cm}$ de diâmetro, colocados sobre camada de algodáo hidrófilo mantida saturada com água destilada em placa de Petri (15 cm de diâmetro). Para evitar a fuga de ácaros, manteve-se a borda da folha coberta com algodáo umedecido. Ninfas e adultos de T. urticae e pólen de mamona, Ricinus communis $\mathrm{L}$., foram colocados em abundância em cada arena, para servir de alimento aos ácaros predadores. A finalidade da transferência dos predadores para arenas de folha de laranjeira foi facilitar a criação e favorecer a conduçáo dos experimentos em laboratório.

Euseius concordis - As populações de E. concordis utilizadas neste trabalho foram coletadas de cultivos de citros, nos municípios de Jaboticabal e Descalvado (SP). Foram transferidos ácaros para folhas de laranjeira da variedade Pera, colocadas sobre camada de algodáo hidrófilo em placa de Petri (15 cm de diâmetro). Para evitar a fuga de ácaros manteve-se a borda da folha coberta com algodáo umedecido. Ninfas e adultos de B. phoenicis e pólen de mamona ou de taboa Typha sp. foram colocados em abundância em cada arena para servir de alimento aos ácaros predadores (ReIs e Sousa, 2001). O pólen de taboa e o de mamona foi armazenado por até 30 dias sob refrigeração $\left(4^{\circ} \mathrm{C}\right)$. 
As criaçóes de E. concordis, assim como de $N$. californicus e B. phoenicis, foram mantidas em salas climatizadas a $25 \pm$ $2^{\circ} \mathrm{C}$, umidade relativa de $70 \pm 10 \%$ e fotofase de 12 horas.

\section{Obtenção das curvas de concentração-mor- talidade}

Arenas de folhas de citros, contendo fêmeas de $N$. californicus ou E. concordis, foram submetidas a pulverizaçôes por meio de torre de Potter (Burkard Scientific, Uxbridge, UK), calibrada a $68,9 \mathrm{kPa}$. Utilizou-se um volume de 2 $\mathrm{mL}$ de calda, em cada pulverização, obtendo-se uma deposição média de resíduo úmido de $1,6 \mathrm{mg} \mathrm{cm}^{-2} \mathrm{da}$ arena. Foram utilizadas 25 fêmeas adultas de cada espécie, com idades de aproximadamente 5 dias, dispostas sobre discos de folha ( $4 \mathrm{~cm}$ de diâmetro), colocados sobre uma camada de algodão hidrófilo em placa de Petri $(9 \mathrm{~cm}$ de diâmetro). A camada de algodáo foi mantida sempre saturada com água destilada. A borda da folha também foi coberta por algodáo úmido, formando uma barreira para evitar a fuga dos ácaros. Foram oferecidos ninfas e adultos de T. urticae em cada arena para servir de alimento ao $N$. californicus. Como fonte de alimento ao ácaro E. concordis foi oferecido pólen de mamona ou de taboa. Após a aplicação, as arenas foram mantidas em câmara climatizada a $25 \pm 2{ }^{\circ} \mathrm{C}, 70 \pm$ $10 \%$ de umidade relativa e fotofase de 12 horas.

Os mesmos procedimentos adotados anteriormente foram usados para os ensaios com os ácaros da leprose, para obtenção das curvas de concentração-mortalidade; entretanto, foram utilizados frutos em lugar das folhas de citros, para evitar a fuga desses ácaros. Os frutos foram lavados com água e, após a secagem, parafinados, deixando-se uma arena de aproximadamente $6 \mathrm{~cm}^{2}$ circundada com cola adesiva (Tanglefoot ${ }^{\circ}$ ) para contê-los.

Foram preparadas quatro a seis concentraçóes de cada produto, através de diluição seqüencial em água destilada, para a obtenção das curvas de concentração-mortalidade e estimativa das concentraçóes letais médias $\left(\mathrm{CL}_{50}\right)$. Os seguintes produtos foram utilizados: abamectina (Vertimec ${ }^{\hat{a}}$ $18 \mathrm{CE}$ ), acefato (Orthene $\left.\mathrm{e}^{\hat{a}} 750 \mathrm{BR}\right)$, azociclotina (Caligur ${ }^{\hat{a}}$ 500 SC), bifentrina (Talstar $\left.{ }^{\hat{a}} 100 \mathrm{CE}\right)$, clorfenapir (Citrex ${ }^{\hat{a}}$ 240), dicofol (Kelthane $\left.e^{\hat{a}} 480 \mathrm{CE}\right)$, dinocape (Karathane $\mathrm{a}^{\hat{a}}$ 369 CE), óxido de fembutatina (Torque ${ }^{\hat{a}} 500$ SC), propargito (Omite $720 \mathrm{CE})$, piridabem (Sanmitề $200 \mathrm{CE}$ ).

A avaliação do número de ácaros vivos e mortos foi realizada 72 horas após a aplicação de cada produto, considerando mortos os ácaros que não conseguiam se locomover por uma distância mínima equivalente ao comprimento do seu corpo, ao serem tocados com um pincel de pelo macio.

Os experimentos foram repetidos pelo menos três vezes. Os resultados foram submetidos à análise de Probit (FInNey, 1971), utilizando-se o programa POLO-PC (Leora Software, 1987). A toxicidade diferencial de cada agrotóxico foi calculada por meio do quociente entre a $\mathrm{CL}_{50}$ do produto para $N$. californicus ou E. concordis e a $\mathrm{CL}_{50}$ para B. phoenicis.

\section{RESULTADOS E DISCUSSÃO}

Os valores de $\mathrm{CL}_{50}, \mathrm{CL}_{90}$ e coeficiente angular obtidos para $B$. phoenicis (Tabela 1), assim como para os predadores $N$.

Tabela 1. Concentração letal (CL) para Brevipalpus phoenicis: $\mathrm{CL}_{50}$ e $\mathrm{CL}_{90}$, intervalos de confiança (I.C.) a $95 \%$, coeficiente angular e erro-padrão da média (EPM); Qui-quadrado $\left(\chi^{2}\right)$; graus de liberdade (G.L.)

\begin{tabular}{|c|c|c|c|c|c|c|c|}
\hline Produto técnico & $\begin{array}{l}\text { Concentração } \\
\text { recomendada } \\
\text { mg L }^{-1} \text { de i.a. }\end{array}$ & $N^{*}$ & $\begin{array}{c}\mathrm{CL}_{50} \\
{[95 \% \text { I.C.] }} \\
\text { mg. } \mathrm{L}^{-1} \text { de i.a. }\end{array}$ & $\begin{array}{c}\mathrm{CL}_{90} \\
{[95 \% \text { I.C.] }} \\
\text { mg. } \mathrm{L}^{-1} \text { de i.a. }\end{array}$ & $\begin{array}{l}\text { Coeficiente angular } \\
\pm \text { EPM }\end{array}$ & $\chi^{2}$ & G.L. \\
\hline Abamectina & 5,4 & 300 & $\begin{array}{c}0,054 \\
(0,054-0,072)\end{array}$ & $\begin{array}{c}0,36 \\
(0,25-0,59)\end{array}$ & $1,65 \pm 0,079$ & 3,335 & 2 \\
\hline Acefato & 750,0 & 340 & $>144.000$ & $>144.000$ & - & - & - \\
\hline Azociclotina & 250,0 & 300 & $\begin{array}{c}10,00 \\
(6,02-32,50)\end{array}$ & $\begin{array}{c}86,00 \\
(28,50-632)\end{array}$ & $1,37 \pm 0,141$ & 0,076 & 2 \\
\hline Bifentrina & 20,0 & 375 & $\begin{array}{c}14,99 \\
(12,62-18,13)\end{array}$ & $\begin{array}{c}69,00 \\
(49,73-110,00)\end{array}$ & $1,93 \pm 0,085$ & 0,431 & 3 \\
\hline Clorfenapir & 150,0 & 425 & $\begin{array}{c}7,44 \\
(6,48-8,64)\end{array}$ & $\begin{array}{c}28,32 \\
(22,08-39,60)\end{array}$ & $2,20 \pm 0,073$ & 5,477 & 4 \\
\hline Dicofol & 370,0 & 375 & $\begin{array}{c}52,32 \\
(39,84-79,68)\end{array}$ & $\begin{array}{c}279,84 \\
(156,96-735,36)\end{array}$ & $1,72 \pm 0,105$ & 3,128 & 3 \\
\hline Dinocape & 184,5 & 375 & $\begin{array}{c}8,85 \\
(6,64-13,65)\end{array}$ & $\begin{array}{c}60,88 \\
(32,47-176,01)\end{array}$ & $1,53 \pm 0,092$ & 6,753 & 3 \\
\hline Óxido de fembutatina & 400,0 & 375 & $\begin{array}{c}17,50 \\
(15,50-20,00)\end{array}$ & $\begin{array}{c}49,50 \\
(40,50-64,50)\end{array}$ & $2,86 \pm 0,110$ & 6,181 & 3 \\
\hline Propargito & 720,0 & 375 & $\begin{array}{c}65,52 \\
(59,04-74,16)\end{array}$ & $\begin{array}{c}164,88 \\
(137,52-208,80)\end{array}$ & $3,20 \pm 0,121$ & 5,567 & 3 \\
\hline Piridabem & 150,0 & 375 & $\begin{array}{c}1,40 \\
(1,20-1,60)\end{array}$ & $\begin{array}{c}4,60 \\
(3,80-6,20)\end{array}$ & $2,45 \pm 0,098$ & 7,282 & 3 \\
\hline
\end{tabular}

${ }^{*} N=$ números de ácaros utilizados no experimento. 
californicus e E. concordis (ambas populaçóes) (Tabela 2, $3 \mathrm{e}$ 4 ), variaram entre os diferentes tratamentos. O coeficiente angular, referente à inclinação da curva de concentraçãoresposta do agrotóxico, serve de indicativo para o grau de homogeneidade da população, e quanto maior seu valor, maior a homogeneidade da população. Nesse aspecto, as populaçôes de E. concordis de Descalvado e Jaboticabal foram mais homogêneas, quanto à sensibilidade à maioria dos acaricidas testados (bifentrina, dicofol, dinocape, óxido de fembutatina, propargito e piribabem), que a população de $N$. californicus. AHMAD et al. (1995) sugerem que a ocorrência de coeficientes angulares com valores inferiores a 2 é um indicativo de heterogeneidade nas populaçóes.

Os valores relativamente baixos de qui-quadrado $\left(\chi^{2}\right)$, observados nas tabelas (1, 2, 3 e 4), indicam que as curvas de concentração-mortalidade, apresentadas nessas tabelas, seguem o modelo de Probit (FInNeY, 1971), baseando-se no número de graus de liberdade.

Tabela 2. Concentração letal (CL) para Neoseiulus californicus: $\mathrm{CL}_{50}$ e $\mathrm{CL}_{90}$, intervalos de confiança (I.C.) a 95\%, coeficiente angular e erro padrão da média (EPM); Qui-quadrado $\left(\chi^{2}\right)$; graus de liberdade (G.L.)

\begin{tabular}{|c|c|c|c|c|c|c|c|}
\hline Produto técnico & $\begin{array}{l}\text { Concentração } \\
\text { recomendada } \\
\text { (mg L-1 de i.a.) }\end{array}$ & $N^{*}$ & $\begin{array}{c}\mathrm{CL}_{50} \\
{[95 \% \text { I.C.] }} \\
\text { (mg L'-1 de i. a.) }\end{array}$ & $\begin{array}{c}\mathrm{CL}_{90} \\
{[95 \% \text { I.C.] }} \\
\text { (mg L'-1 de i. a.) }\end{array}$ & $\begin{array}{c}\text { Coeficiente } \\
\text { angular } \pm \text { EPM }\end{array}$ & $\chi^{2}$ & G.L. \\
\hline Abamectina & 5,4 & 360 & $\begin{array}{c}2,82 \\
(2,23-3,78)\end{array}$ & $\begin{array}{c}15,55 \\
(9,88-31,03)\end{array}$ & $1,73 \pm 0,082$ & 5,027 & 4 \\
\hline Acefato & 750,0 & 360 & $>144.000$ & $>144.000$ & - & - & - \\
\hline Azociclotina & 250,0 & 360 & $\begin{array}{c}208,50 \\
(162,5-292,50)\end{array}$ & $\begin{array}{c}1.129,50 \\
(681,50-2.468,00)\end{array}$ & $1,74 \pm 0,082$ & 1,845 & 4 \\
\hline Bifentrina & 20,0 & 300 & $\begin{array}{c}459,26 \\
(356,91-617,13)\end{array}$ & $\begin{array}{c}3.853,83 \\
(2.205,03-9.831,97)\end{array}$ & $1,38 \pm 0,085$ & 0,929 & 3 \\
\hline Clorfenapir & 150,0 & 360 & $\begin{array}{c}55,44 \\
(45,36-75,60)\end{array}$ & $\begin{array}{c}204,24 \\
(131,04-434,88)\end{array}$ & $2,26 \pm 0,126$ & 0,831 & 4 \\
\hline Dicofol & 370,0 & 300 & $\begin{array}{c}78,72 \\
(62,40-107,04)\end{array}$ & $\begin{array}{c}384,48 \\
(238,56-829,44)\end{array}$ & $1,85 \pm 0,106$ & 2,909 & 3 \\
\hline Dinocape & 184,5 & 300 & $\begin{array}{c}1.249,77 \\
(945,64-1.843,38)\end{array}$ & $\begin{array}{c}12.290,25 \\
(6.391,83-35.084,81)\end{array}$ & $1,29 \pm 0,071$ & 7,188 & 3 \\
\hline Óxido de fembutatina & 400,0 & 240 & $\begin{array}{c}9.298,50 \\
(6.889,00-13.309,50)\end{array}$ & $\begin{array}{c}113.143,50 \\
(61.727,50-277.018,50)\end{array}$ & $1,18 \pm 0,051$ & 1,209 & 2 \\
\hline Propargito & 720,0 & 300 & $\begin{array}{c}4.350,24 \\
(3.125,52-7.560,72)\end{array}$ & $\begin{array}{c}2.2767,85 \\
(11.602,08-79.312,32)\end{array}$ & $1,78 \pm 0,126$ & 1,675 & 3 \\
\hline Piridabem & 150,0 & 300 & $\begin{array}{c}12,40 \\
(9,20-20,20)\end{array}$ & $\begin{array}{c}76,20 \\
(39,60-242,20)\end{array}$ & $1,63 \pm 0,107$ & 5,645 & 3 \\
\hline
\end{tabular}

${ }^{*} N=$ números de ácaros utilizados no experimento.

Tabela 3. Concentração letal (CL) para Euseius concordis coletado no município de Descalvado: $\mathrm{CL}_{50}$ e $\mathrm{CL}_{90}$, intervalos de confiança (I.C.) a $95 \%$, coeficiente angular e erro-padrão da média (EPM); Qui-quadrado $\left(\chi^{2}\right)$; graus de liberdade (G.L.)

\begin{tabular}{|c|c|c|c|c|c|c|c|}
\hline Produto técnico & $\begin{array}{l}\text { Concentração } \\
\text { recomendada } \\
\text { (mg L-1 de i. a.) }\end{array}$ & $N^{*}$ & $\begin{array}{c}\mathrm{CL}_{50} \\
{[95 \% \text { I.C. }]}\end{array}$ & $\begin{array}{c}\mathrm{CL}_{90} \\
{[95 \% \text { I.C.] }} \\
\text { (mg L'-1 de i. a.) }\end{array}$ & $\begin{array}{c}\text { Coeficiente } \\
\text { angular } \pm E P M\end{array}$ & $\chi^{2}$ & G.L. \\
\hline Abamectina & 5,4 & 300 & $\begin{array}{c}4,29 \\
(3,77-4,88)\end{array}$ & $\begin{array}{c}11,10 \\
(9,12-14,56)\end{array}$ & $3,10 \pm 0,149$ & 3,464 & 3 \\
\hline Acefato & 750,0 & 240 & $\begin{array}{c}1,26 \\
(1,05-1,60)\end{array}$ & $\begin{array}{c}4,71 \\
(3,23-8,72)\end{array}$ & $2,24 \pm 0,149$ & 4,525 & 2 \\
\hline Azociclotina & 250,0 & 240 & $\begin{array}{c}42,14 \\
(35,0-53,51)\end{array}$ & $\begin{array}{c}165,49 \\
(112,31-307,45)\end{array}$ & $2,15 \pm 0,135$ & 1,61 & 2 \\
\hline Bifentrina & 20,0 & 300 & $\begin{array}{c}8,84 \\
(7,58-10,28)\end{array}$ & $\begin{array}{c}34,13 \\
(26,95-46,80)\end{array}$ & $2,18 \pm 0,086$ & 1,543 & 3 \\
\hline Clorfenapir & 150,0 & 300 & $\begin{array}{c}29,33 \\
(25,58-33,96)\end{array}$ & $\begin{array}{c}88,77 \\
(70,87-120,29)\end{array}$ & $2,66 \pm 0,106$ & 4,549 & 3 \\
\hline Dicofol & 370,0 & 300 & $\begin{array}{c}127,58 \\
(109,53-149,87)\end{array}$ & $\begin{array}{c}389,71 \\
(306,62-543,74)\end{array}$ & $2,64 \pm 0,115$ & 2,366 & 3 \\
\hline Dinocape & 184,5 & 300 & $\begin{array}{c}133,14 \\
(116,52-152,88)\end{array}$ & $\begin{array}{c}390,72 \\
(317,16-515,19)\end{array}$ & $2,74 \pm 0,105$ & 6,148 & 3 \\
\hline Óxido de fembutatina & 400,0 & 300 & $\begin{array}{c}2.159,75 \\
(1790,05-2.790,05)\end{array}$ & $\begin{array}{c}12.038,69 \\
(8.312,92-20.687,82)\end{array}$ & $1,71 \pm 0,081$ & 1,925 & 3 \\
\hline Propargito & 720,0 & 240 & $\begin{array}{c}172,36 \\
(144,04-201,80)\end{array}$ & $\begin{array}{c}508,17 \\
(407,08-702,86)\end{array}$ & $2,73 \pm 0,162$ & 2,193 & 2 \\
\hline Piridabem & 150,0 & 300 & $\begin{array}{c}34,9 \\
(30,94-39,36)\end{array}$ & $\begin{array}{c}86,02 \\
(72,76-106,78)\end{array}$ & $3,27 \pm 0,121$ & 3,593 & 3 \\
\hline
\end{tabular}

${ }^{*} N=$ números de ácaros utilizados no experimento. 
Os produtos abamectina, azociclotina, clorfenapir, dicofol, dinocape, óxido de fembutatina, propargito e piridabem foram altamente tóxicos a $B$. phoenicis, com $\mathrm{CL}_{90}$ igual ou inferior às suas concentraçôes recomendadas. Bifentrina não foi efetivo como os produtos anteriormente mencionados, com valor de $\mathrm{CL}_{90} 3,45$ vezes maior que a concentração recomendada (Tabela 1). O produto acefato foi o menos tóxico a $B$. phoenicis, com $\mathrm{CL}_{50}$ maior que $144.000 \mathrm{mg} \mathrm{L}^{-1}$ de i.a.Para esse produto, não foi possível estimar as $\mathrm{CL}_{50}$ ou $\mathrm{CL}_{90}$, com seus intervalos de confiança, devido à baixíssima sensibilidade do ácaro ao ingrediente ativo. As concentraçôes necessárias para a sensibilizaçâo do ácaro foram tão elevadas que impossibilitaram a obtenção de uma curva de concentração-mortalidade, através de bioensaio com torre de Potter, devido a problemas de aplicação desuniforme por entupimento do bico de pulverização. A concentração de $144.000 \mathrm{mg} \mathrm{L}^{-1}$ de i.a. é 192 vezes maior que a concentraçáo recomendada de acefato para citros no Brasil (AGROFIT, 2009).

O ácaro $N$. californicus possui baixa sensibilidade a acefato, bifentrina, dinocape, óxido de fembutatina e propargito, com valores de $\mathrm{CL}_{50}$ pelo menos seis vezes maiores que as concentraçōes recomendadas dos produtos (Tabela 2). No caso de acefato, da mesma forma que para $B$. phoenicis, não foi possível estimar as $\mathrm{CL}_{50}$ e $\mathrm{CL}_{90}$, com seus intervalos de confiança, devido à necessidade de uso de concentraçóes extremamente elevadas para se obter alguma mortalidade em $N$. californicus. Concentraçôes acima de $144.000 \mathrm{mg} \mathrm{L}^{-1}$ de i.a. causaram baixíssimas mortalidades (<10\%) no ácaro predador.

A espécie $N$. californicus também foi altamente tolerante a óxido de fembutatina e bifentrina, com valores de $\mathrm{CL}_{50}$ iguais ou superiores a concentraçôes 22 vezes maiores que as recomendadas. As $\mathrm{CL}_{90}$ de óxido de fembutatina $\mathrm{e}$ bifentrina foram, respectivamente, 282 e 193 vezes maiores que suas concentraçôes recomendadas (AGROFIT, 2009). A concentraçáo letal média de dinocape para $N$. californicus foi de 1.249,77 $\mathrm{mg} \mathrm{L}^{-1}$ de i.a., que corresponde à aproximadamente sete vezes a concentração recomendada deste acaricida para o controle de B. phoenicis em citros (AGROFIT, 2009). A baixa sensibilidade de $N$. californicus a bifentrina, dinocape, óxido de fembutatina e propargito já havia sido reportada por Silva e Oliveira (2006; 2007).

Embora os piretróides, de modo geral, tenham alta toxicidade aos ácaros fitoseídeos E. concordis, Euseius citrifolius Denmark \& Muma, I. zuluagai, P. persimilis e T. pyri (Zacharda e Hlùchy, I99I; Malezieux et al., I992; Sato et al., 1994; 1996), essa população de N. californicus, coletada de cultivo comercial de morangueiro, tem sido pouco sensível a este grupo químico (SATO et al., 2002; Silva e Oliveira, 2006; 2007). Easterbrook et al. (2001) também mencionam a baixa sensibilidade de $N$. californicus a piretróides o que pode indicar tolerância natural da espécie a este grupo químico.

Abamectina, azociclotina, clorfenapir, dicofol e piridabem foram tóxicos a $N$. californicus, com valores de $\mathrm{CL}_{50}$ iguais ou inferiores às suas concentraçóes recomendadas (Tabela 2). As menores diferenças entre as concentraçôes recomendadas e a $\mathrm{CL}_{50}$ dos produtos foram observadas para azociclotina e abamectina ( $<2$ vezes). As maiores diferenças foram observadas para dicofol e piridabem, para os quais, as $\mathrm{CL}_{50}$ foram, respectivamente, 4,7 e 12,0 vezes menores que as concentraçoóes recomendadas.

Segundo Silva e Oliveira (2006), o acaricida azociclotina, na sua concentração recomendada, foi considerado

Tabela 4. Concentração letal (CL) para Euseius concordis coletado no município de Jaboticabal: $\mathrm{CL}_{50} \mathrm{e} \mathrm{CL}_{90}$, intervalos de confiança (I.C.) a $95 \%$, coeficiente angular e erro padrão da média (EPM); Qui-quadrado $\left(\chi^{2}\right)$; graus de liberdade (G.L.)

\begin{tabular}{|c|c|c|c|c|c|c|c|}
\hline Produto técnico & $\begin{array}{l}\text { Concentração } \\
\text { recomendada } \\
\text { (mg L-1 de i.a.) }\end{array}$ & $N^{*}$ & $\begin{array}{c}\mathrm{CL}_{50} \\
\text { (mg de i. a.L-1) } \\
{[95 \% \text { I.C.] }}\end{array}$ & $\begin{array}{c}\mathrm{CL}_{90} \\
\text { (mg de i. a.L.-1) } \\
{[95 \% \text { I.C.] }}\end{array}$ & $\begin{array}{c}\text { Coeficiente } \\
\text { angular } \pm \text { EPM }\end{array}$ & $\chi^{2}$ & G.L. \\
\hline Abamectina & 5,4 & 300 & $\begin{array}{c}3,23 \\
(2,63-4,02)\end{array}$ & $\begin{array}{c}17,48 \\
(11,92-31,18)\end{array}$ & $1,74 \pm 0,090$ & 1,168 & 3 \\
\hline Acefato & 750,0 & 240 & $\begin{array}{c}1,34 \\
(1,12-1,69)\end{array}$ & $\begin{array}{c}4,70 \\
(3,27-8,49)\end{array}$ & $2,36 \pm 0.155$ & 4,315 & 2 \\
\hline Azociclotina & 250,0 & 240 & $\begin{array}{c}333,0 \\
(206,90-432,25)\end{array}$ & $\begin{array}{c}2.864,90 \\
(925,63-69.562,06)\end{array}$ & $1,37 \pm 0,169$ & 0,979 & 2 \\
\hline Bifentrina & 20,0 & 360 & $\begin{array}{c}151,42 \\
(123,14-188,60)\end{array}$ & $\begin{array}{c}921,35 \\
(634,94-1.595,57)\end{array}$ & $1,63 \pm 0,066$ & 2,719 & 4 \\
\hline Clorfenapir & 150,0 & 300 & $\begin{array}{c}73,20 \\
(61,12-89,01)\end{array}$ & $\begin{array}{c}292,36 \\
(213,94-459,91)\end{array}$ & $2,13 \pm 0,102$ & 7,194 & 3 \\
\hline Dicofol & 370,0 & 300 & $\begin{array}{c}409,52 \\
(352,25-477,10)\end{array}$ & $\begin{array}{c}1.217,55 \\
(974,12-1.647,84)\end{array}$ & $2,70 \pm 0,114$ & 5,141 & 3 \\
\hline Dinocape & 184,5 & 240 & $\begin{array}{c}162,10 \\
(126,56-247,95)\end{array}$ & $\begin{array}{c}652,12 \\
(374,63-1.975,22)\end{array}$ & $2,12 \pm 0,186$ & 5,130 & 2 \\
\hline Óxido de fembutatina & 400,0 & 300 & $\begin{array}{c}2.342,85 \\
(1.968,78-2832,20)\end{array}$ & $\begin{array}{c}8.948,76 \\
(6.607,12-13.833,85)\end{array}$ & $2,20 \pm 0.102$ & 6,081 & 3 \\
\hline Propargito & 720,0 & 300 & $\begin{array}{c}383,61 \\
(308,39-543,31)\end{array}$ & $\begin{array}{c}1.485,68 \\
(909,72-3.663,30)\end{array}$ & $2,18 \pm 0.152$ & 6,239 & 3 \\
\hline Piridabem & 150,0 & 300 & $\begin{array}{c}22.46 \\
(19,35-26,20)\end{array}$ & $\begin{array}{c}62,21 \\
(52,88-89,90)\end{array}$ & $2,73 \pm 0.115$ & 0,264 & 3 \\
\hline
\end{tabular}

${ }^{*} N$ = números de ácaros utilizados no experimento. 
levemente nocivo para $N$. californicus. Os autores também verificaram que resíduos do agrotóxico em folhas de citros provocaram mortalidades de até $24 \%$ no ácaro predador. $\mathrm{O}$ efeito tóxico do produto permaneceu por um período inferior a três dias.

A toxicidade de abamectina a fêmeas adultas de $N$. californicus também foi reportada por SATO et al. (2002). Embora abamectina mostre-se prejudicial a $N$. californicus, tem sido observado curto efeito residual do produto. Sato et al. (2002) e Silva e Oliveira (2007) verificaram que resíduos secos da aplicação de abamectina, na sua concentração recomendada, provocaram mortalidades entre $37,5 \%$ e $29,8 \%$, em $N$. californicus, por um período igual ou inferior a um dia. Resultado semelhante também foi observado para I. zuluagai, para o qual o período residual foi de apenas um dia, com mortalidade inicial inferior a 62\% (SATO et al., 1996). A elevada toxicidade de clorfenapir a $N$. californicus também foi reportada por Sato et al. (2002) e Silva e Oliveira (2007). Segundo esses autores, a toxicidade residual de clorfenapir é mais longa que de abamectina, causando mortalidades iniciais entre $57 \%$ e $100 \%$, na sua concentração recomendada, mantendo um efeito prejudicial significativo por até sete dias após a aplicação. Reis e Sousa (2001) também ressaltaram a alta toxicidade desse agrotóxico aos ácaros fitoseídeos das espécies Euseius alatus DeLeon e I. zuluagai.

Em contraste com $N$. californicus, que foi pouco sensível a diversos produtos, a população de $E$. concordis procedente de Descalvado foi suscetível a quase todos os produtos testados. Apenas óxido de fembutatina foi pouco tóxico ao predador, com valor de $\mathrm{CL}_{50} 5,4$ vezes acima da concentração recomendada. Para os demais produtos, a $\mathrm{CL}_{50}$ foi igual ou inferior à recomendada (Tabela 3). A baixa toxicidade de óxido de fembutatina em ácaros do gênero Euseius também foi observada por SATO et al. (1994), que mencionaram mortalidades de adultos de $E$. citrifolius inferiores a $20 \%$, quando expostos aos resíduos tóxicos do acaricida, pulverizado na sua concentração recomendada sobre folhas de citros.

Ao contrário de $N$. californicus e $B$. phoenicis, altamente tolerantes a acefato, as duas populaçôes de E. concordis (Descalvado e Jaboticabal) foram extremamente sensíveis a esse acaricida. $\mathrm{A} \mathrm{CL}_{50}$ de acefato para $E$. concordis foi pelo menos 550 vezes menor que a concentração recomendada do produto para citros (Tabelas 3 e 4). Comparando-se as duas populaçôes de E. concordis (Tabelas 3, 4 e 5), observa-se que a populaçấo de Jaboticabal é mais resistente que a populaçáo de Descalvado a diversos produtos, tais como azociclotina, bifentrina, clorfenapir, dicofol e propargito, baseando-se na não-sobreposição dos intervalos de confiança $\mathrm{da} \mathrm{CL}_{50}$. Os maiores contrastes foram observados para bifentrina e azociclotina, para os quais, a população de Jaboticabal foi, respectivamente, 17,1 e 7,9 vezes mais resistente que a de Descalvado (Tabela 5). Essas diferenças de sensibilidade estão provavelmente associadas às diferenças nos regimes de aplicaçôes de produtos químicos nos pomares cítricos dos dois municípios. No pomar de Descalvado, têm sido realizadas poucas aplicaçóes de acaricidas, evitando-se a aplicação do mesmo acaricida no mesmo ano agrícola, realizando-se ainda a rotaçáo de princípios ativos para retardar a evolução da resistência. A estratégia de manejo adotado em Descalvado, visando à manutenção da suscetibilidade das populações de B. phoenicis a acaricidas, pode ter influenciado na sensibilidade de $E$. concordis aos acaricidas testados.

Tabela 5. Testes toxicológicos com agrotóxicos: concentração letal média $\left(\mathrm{CL}_{50}\right)$ e toxicidade diferencial (TD)

\begin{tabular}{|c|c|c|c|c|c|c|c|}
\hline \multirow[t]{2}{*}{ Agrotóxicos } & \multicolumn{2}{|l|}{ N. californicus } & \multicolumn{2}{|c|}{$\begin{array}{l}\text { E. concordis } \\
\text { Descalvado }\end{array}$} & \multicolumn{2}{|c|}{$\begin{array}{l}\text { E. concordis } \\
\text { Jaboticabal }\end{array}$} & \multirow{2}{*}{$\begin{array}{c}\text { B. phoenicis } \\
\mathrm{CL}_{50}\end{array}$} \\
\hline & $\mathrm{CL}_{50}$ & TD* & $\mathrm{CL}_{50}$ & TD* & $\mathrm{CL}_{50}$ & TD* & \\
\hline Abamectina & $\begin{array}{c}2,82 \\
(2,23-3,78)\end{array}$ & 52 & $\begin{array}{c}4,29 \\
(3,77-4,88)\end{array}$ & 79 & $\begin{array}{c}3,23 \\
(2,63-4,02)\end{array}$ & 60 & $\begin{array}{c}0,054 \\
(0,054-0,072)\end{array}$ \\
\hline Acefato & $>144.000$ & $\sim 1$ & $\begin{array}{c}1,26 \\
(1,05-1,60)\end{array}$ & $<0,0000087$ & $\begin{array}{c}1,34 \\
(1,12-1,69)\end{array}$ & $<0,0000093$ & $>144.000$ \\
\hline Azociclotina & $\begin{array}{c}208,50 \\
(162,50-292,50)\end{array}$ & 20,85 & $\begin{array}{c}42,14 \\
(35,0-53,51)\end{array}$ & 4,21 & $\begin{array}{c}333,01 \\
(206,90-432,25)\end{array}$ & 33,30 & $\begin{array}{c}10,00 \\
(6,02-32,50)\end{array}$ \\
\hline Bifentrina & $\begin{array}{c}459,26 \\
(356,91-617,13)\end{array}$ & 30,63 & $\begin{array}{c}8,84 \\
(7,58-10,28)\end{array}$ & 0,59 & $\begin{array}{c}151,42 \\
(123,14-188,60)\end{array}$ & 10,10 & $\begin{array}{c}14,99 \\
(12,62-18,13)\end{array}$ \\
\hline Clorfenapir & $\begin{array}{c}55,44 \\
(45,36-75,60)\end{array}$ & 7,45 & $\begin{array}{c}29,33 \\
(25,58-33,96)\end{array}$ & 3,94 & $\begin{array}{c}73,20 \\
(61,12-89,01)\end{array}$ & 9,83 & $\begin{array}{c}7,44 \\
(6,48-8,64)\end{array}$ \\
\hline Dicofol & $\begin{array}{c}78,72 \\
(62,40-107,04)\end{array}$ & 1,50 & $\begin{array}{c}127,58 \\
(109,53-149,87)\end{array}$ & 2,43 & $\begin{array}{c}409,44 \\
(352,25-477,10)\end{array}$ & 7,82 & $\begin{array}{c}52,32 \\
(39,84-79,68)\end{array}$ \\
\hline Dinocape & $\begin{array}{c}1249,77 \\
(945,64-1843,38)\end{array}$ & 141 & $\begin{array}{c}133,14 \\
(116,52-152,88)\end{array}$ & 14 & $\begin{array}{c}162,10 \\
(126,56-247,95)\end{array}$ & 17 & $\begin{array}{c}8,85 \\
(6,64-13,65)\end{array}$ \\
\hline Óxido de fembutatina & $\begin{array}{c}9298,50 \\
(6889,00-13309,50)\end{array}$ & 531 & $\begin{array}{c}2159,75 \\
(1790,05-2790,05)\end{array}$ & 123 & $\begin{array}{c}2342,85 \\
(1968,78-2832,20)\end{array}$ & 134 & $\begin{array}{c}17,50 \\
(15,50-20,00)\end{array}$ \\
\hline Propargito & $\begin{array}{c}4350,24 \\
(3125,52-7560,72)\end{array}$ & 66 & $\begin{array}{c}172,36 \\
(144,04-201,80)\end{array}$ & 2,63 & $\begin{array}{c}383,61 \\
(308,39-543,31)\end{array}$ & 5,85 & $\begin{array}{c}65,52 \\
(59,04-74,16)\end{array}$ \\
\hline Piridabem & $\begin{array}{c}12,40 \\
(9,20-20,20)\end{array}$ & 8,85 & $\begin{array}{c}34,90 \\
(30,94-39,36)\end{array}$ & 25 & $\begin{array}{c}22,46 \\
(19,35-26,20)\end{array}$ & 16 & $\begin{array}{c}1,40 \\
(1,20-1,60)\end{array}$ \\
\hline
\end{tabular}

* Toxicidade diferencial $=\mathrm{CL}_{50}$ para fitoseídeos dividido pela $\mathrm{CL}_{50}$ para $B$. phoenicis. 
Embora PolETti (2002) não tenha observado variabilidade intraespecífica na suscetibilidade de $E$. concordis a dicofol, ao avaliar três populaçôes do ácaro predador (de Presidente Prudente, Juazeiro e Limeira), verificou-se no presente trabalho que a população de E. concordis de Jaboticabal foi 3,2 vezes mais resistente que a de Descalvado, baseando-se nas $\mathrm{CL}_{50}$ das duas populaçóes (Tabelas $3 \mathrm{e} 4$ ). $\mathrm{Na}$ populaçáo de Descalvado constatou-se $\mathrm{CL}_{50}[127,6$ (I.C.: 109,5 a $149,9 \mathrm{mg} \mathrm{L}^{-1}$ de i.a.)] semelhante à mencionada por Polettri (2002) para a população coletada de pomar cítrico de Presidente Prudente (população Ec-1, da Estaçáo Experimental do Instituto Biológico) $\left[\mathrm{CL}_{50}=\right.$ 109,3 (I.C.: 71,6 - 144,4 $\mathrm{mg} \mathrm{L}^{-1}$ de i.a.)].

Comparando-se as $\mathrm{CL}_{50}$ dos produtos testados para $N$. californicus e B. phoenicis, observa-se que o ácaro predador teve tolerância igual ou superior à do ácaro praga a todos os produtos testados. As toxicidades diferenciais $\left(\mathrm{CL}_{50}\right.$ para o fitoseídeo dividido pela $\mathrm{CL}_{50}$ para B. phoenicis) foram superiores a 20 vezes para os produtos abamectina, azociclotina, bifentrina, propargito, dinocape e óxido de fembutatina. Para os dois últimos produtos, as toxicidades diferenciais foram acima de 140 vezes (Tabela 5).

Embora $N$. californicus seja menos sensível a piridabem que B. phoenicis (Tabela 5), a concentração registrada para o controle do ácaro da leprose (150 $\mathrm{mg} \mathrm{L}^{-1}$ de i.a.) (Agrofit, 2009) é 12 vezes maior que a concentração letal média obtida para o predador. Essa menor sensibilidade do predador em relaçáo ao ácaro-praga pode ser vantajosa no restabelecimento populacional do predador após a aplicação do acaricida.

A população de E. concordis de Descalvado foi mais tolerante que $B$. phoenicis à maioria dos produtos testados, porém com valores de toxicidade diferencial (TD) geralmente inferiores às apresentadas por $N$. californicus. Os únicos casos em que as $\mathrm{CL}_{50}$ para E. concordis de Descalvado foram maiores que os observados para $N$. californicus foram para dicofol e piridabem. Para esses produtos as diferenças entre as $\mathrm{CL}_{50}$ para essas populaçōes foram iguais ou inferiores a 2,8 vezes (Tabela 5 ). A maior diferença entre as populaçốes de $N$. californicus e de E. concordis de Descalvado foi observada para acefato, para o qual $N$. californicus mostrou-se pelo menos 114 mil vezes mais tolerante que $E$. concordis. Essa diferença de $N$. californicus em relação a $E$. concordis foi um pouco menor em relação à populaçáo de Jaboticabal, porém, ainda acima de 107 mil vezes (Tabela 5). Consideráveis diferenças entre as populaçóes de N. californicus e E. concordis de Descalvado também foram observadas para bifentrina e propargite, para os quais $N$. californicus foi, respectivamente, 51,9 e 25,2 vezes mais tolerante.

A população de E. concordis de Jaboticabal também foi mais tolerante que a de $B$. phoenicis a quase todos os produtos testados, com toxicidades diferenciais oscilando entre 5,85 e 134 vezes. A única exceção foi acefato, para o qual, a população de Jaboticabal foi bem mais sensível que o ácaro-praga. Os maiores contrastes entre as populaçôes de E. concordis de Jaboticabal e B. phoenicis, sem considerar acefato, foram observados para óxido de fembutatina e abamectina, para os quais as toxicidades diferenciais foram, respectivamente, de 134 e 60 vezes, (Tabela 5). Esses dois produtos foram utilizados várias vezes no pomar cítrico de Jaboticabal visando ao controle de ácaros-praga e no caso específico de abamectina, visando também ao minador dos citros, Phyllocnistis citrella Stainton (Lepidoptera: Gracillariidae).

Em relação a $N$. californicus, a população de $E$. concordis de Jaboticabal foi mais tolerante a dicofol (5,2 vezes), e com tolerância semelhante aos produtos abamectina, azociclotina, clorfenapir e piridabem. Para os demais produtos (acefato, bifentrina, dinocape, óxido de fembutatina e propargito), N. californicus foi mais tolerante. Essa populaçáo de E. concordis de Jaboticabal, assim como a de $N$. californicus, é bastante interessante para programas de manejo integrado de pragas, devido a sua alta tolerância a diversos agrotóxicos, incluindo piretróides (ex.: bifentrina) que, normalmente, são prejudiciais a ácaros predadores da família Phytoseiidae (Malezieux et al., I992; SATO et al., 1994; 1996).

Poletti (2002) detectou resistência à deltametrina em populaçôes de E. concordis coletadas em diferentes pomares de laranjeiras no Estado de São Paulo, e observou que a resistência esteve associada ao histórico de pulverizaçóes com o inseticida nos locais onde foram efetuadas as coletas. Outro exemplo de ácaros fitoseídeos que também possuem diferenças de tolerância a piretróides foi reportado para T. pyri, que foi mais tolerante a cipermetrina, deltametrina e fenvalerato que $P$. persimilis. Com relação à deltametrina, a razáo de tolerância observada para essas duas espécies atingiu valores de aproximadamente 314 vezes (MARWICK, 1986).

Em países da Europa e nos EUA, programas de manejo integrado têm se utilizado populaçôes de ácaros fitoseídeos resistentes evoluídas no campo, assim como selecionadas em laboratório (Hoy, I985, Hoy e Ouyang, I989; HardMAM et al., 2000). O uso desses inimigos naturais resistentes é parte integrante do manejo de ácaros fitófagos em diversas culturas, incluindo maçã (Blommers, 1994), amêndoas (Hoy, 1985) e plantas em casas de vegetaçáo (Hussey e SCOPES, 1985).

Apesar do elevado número de casos de programas de manejo de pragas com base na liberaçâo de linhagens resistentes de inimigos naturais (destacando-se os ácaros fitoseídeos) em vários países, no Brasil esse assunto ainda é incipiente. No entanto, as possibilidades de exploração desta ferramenta, que agrega harmoniosamente o controle químico ao biológico, parecem promissoras (MONTEIRO, 2002). Em citros, estudos revelaram que a evoluçáo para a resistência ao inseticida deltametrina em algumas populaçóes de ácaros fitoseídeos pode estar sendo responsável pela permanência desses ácaros em algumas áreas, 
contribuindo dessa forma para o controle biológico efetivo de ácaros-praga como B. phoenicis (Poletti, 2002).

No caso do emprego de $N$. californicus para o controle de $B$. phoenicis, a utilização de linhagens resistentes desse predador poderia contribuir para a preservação contínua dessa espécie nos pomares, inclusive durante as pulverizaçóes para o controle de insetos e ácaros comumente associados a essa cultura. A introdução de populaçôes de fitoseídeos tolerantes ou resistentes a produtos químicos, como as observadas neste estudo, em agroecossistemas citrícolas, possibilitaria a manutenção de um ambiente favorável ao controle biológico exercido pelos predadores, mesmo diante da aplicação de agrotóxicos, sem que houvesse mortalidade destes inimigos naturais, contribuindo para minimizar os danos provocados por pragas na cultura. A redução no uso de acaricidas e, consequentemente, dos custos de produçáo e da quantidade de resíduos no produto final, atenderia melhor às imposiçóes de um mercado que vem se tornando a cada dia mais exigente.

\section{CONCLUSÕES}

1. Abamectina, azociclotina, clorfenapir, dicofol, dinocape, óxido de fembutatina, propargito e piridabem são altamente tóxicos a $B$. phoenicis, com valores de $\mathrm{CL}_{90}$ iguais ou inferiores às concentraçôes recomendas desses produtos.

2. O predador $N$. californicus é mais tolerante aos agrotóxicos bifentrina, propargito, óxido de fembutatina e dinocape que as populaçóes de $B$. phoenicis e $E$. concordis de Descalvado (SP) e Jaboticabal (SP).

3. A população de E. concordis de Jaboticabal é mais resistente que a de Descalvado a azociclotina, bifentrina, clorfenapir, dicofol e propargito.

4. Acefato é extremamente tóxico a $E$. concordis, mas inócuo a adultos de $N$. californicus.

\section{AGRADECIMENTOS}

Ao Dr. Gilberto José de Moraes (ESALQ/USP) e Dr. Jeferson Luiz de Carvalho Mineiro (Instituto Biológico) pelo auxílio na identificaçáo dos ácaros predadores. À CAPES (Coordenação de Aperfeiçoamento de Pessoal de Nível Superior) pela bolsa de doutorado ao primeiro autor. Ao CNPq pela bolsa de produtividade em pesquisa ao segundo autor.

\section{REFERÊNCIAS}

AGROFIT. Sistema de Agrotóxicos Fitossanitários, do Ministério da Agricultura, Pecuária e Abastecimento. Disponível em: <http:extranet.agricultura.gov.br/agrofit_ cons/principal_agrofit_ cons>. Acesso em: 20 fev. 2009.

AHMAD, M.; ARIF, M.I.; AHMAD, Z. Insecticide resistance in Helicoperva argimera (Lepidoptera: Noctuidae) in Pakistan. Journal of Economic Entomology, v.88, p.771-776, 1995.

BLOMMERS, L.H.M. Integrated pest management in European apple orchards. Annual Review of Entomology, v.39, p.213-241, 1994.

CROFT, B.A. Management of pesticide resistance in arthropod pests. In: GREEN, M.B., MOBERG, W.K.; LEBARON, H. (Ed.). Managing resistance to agrochemicals: fundamental and practical approaches to combating resistance. Washington, DC: American Chemical Society, 1990. p.149-168.

DUNLEY, J.E.; MESSING, R.H.; CROFT, B.A. Levels and genetics of organophosphate resistance in Italian and Oregon biotypes of Amblyseius andersoni (Acari: Phytoseiidae). Journal of Economic Entomology, v.84, p.750-755, 1991.

EASTERBROOK, M.A.; FITZGERALD, J.D.; SOLOMON, M.G. Biological control of strawberry tarsonemid mite Phytonemus pallidus and two-spotted spider mite Tetranychus urticae on strawberry in the UK using species of Neoseiulus (Amblyseius) (Acari: Phytoseiidae). Experimental and Applied Acarology, v.25, p.25-36, 2001.

FINNEY, D.J. Probit analysis. 3.ed. London: Cambridge University Press, 1971.315p.

HARDMAM, J.M.; MOREAU, D.L.; SNYDER, M.; GAUL, S.O.;BENT, E.D. Performance of a pyrethroid-resistent strain of the predador mite Typhlodromus pyri (Acari:Phytoseiidae) under diferent insecticide regimes. Journal of Economic Entomology, v.93, p.590-604, 2000.

HOY, M.A. Recent advances in genetics and genetic improvement of the Phytoseiidae. Annual Review of Entomology, v.30, p.345$370,1985$.

HOY, M.A.; OUYANG, Y.L. Selection of the western predatory mite, Metaseiulus occidentalis (Acari: Phytoseiidae), for resistance to abamectin. Journal of Economic Entomology, v.82, p.35-40, 1989.

HUSSEY, N.W.; SCOPES, N.E.A. Greenhouse vegetables. In: HELLE, W.; SABELIS, M.W. (Ed.). Spider mites: their biology, natural enemies, and control. Amsterdam: Elsevier, 1985. vol. $1 \mathrm{~b}$. p.285-298.

LEORA SOFTWARE. POLO-PC: a user's guide to Probit or Logit analysis. Berkeley, 1987. 20p.

MALEZIEUX, S.L.; Lapchin, M.; Pralavorio, J.C. Toxicity of pesticide residues to beneficial arthropod, Phytoseiulus persimilis (Acari: Phytoseiidae). Journal of Economic Entomology, v.85, p.2077-2081, 1992.

MARWICK, N.P. Detecting variability and selecting for pesticide resistance into two species of Phytoseiidae mites. Entomophaga, v.31, p.225-236, 1986. 
MONTEIRO, L.B. Manejo integrado de pragas em macieira no Rio Grande do Sul II. Uso de Neoseiulus californicus para controle de Panonychus ulmi. Revista Brasileira de Fruticultura, v.24, p.395405, 2002.

NEVES, E.M.; RODRIGUES, L.; GASTALDI, H.L.G. Defensivos agrícolas e custos na produção de citros. Visão Agrícola, v.1, p.127$131,2004$.

OMOTO, C.; ALVES, E.B.; RIBEIRO, P.C. Detecção e monitoramento da resistência de Brevipalpus phoenicis (Geijskes) (Acari: Tenuipalpidae) ao dicofol. Anais da Sociedade Entomológica do Brasil, v.29, p.757-764, 2000.

PENMAN, D.R.; WEARING, C.H.; COLYER, E.; THOMAS, W.P. The role of insecticide-resistant phytoseiids in integrated mite control in New Zealand. In J.G. RODRIGUES (Ed.). Recent advances in acarology, New York, Academic Press, 1979. Vol. 1. p.59-69.

POLETTI, M. Variabilidade inter e intraespecífica na suscetibilidade de ácaros fitoseídeos (Acari: Phytoseiidae) a dicofol e deltametrina em citros. 78f. 2002. Dissertação (Mestrado), Escola Superior de Agricultura “Luiz de Queiroz” USP.

REIS, P.R.; SOUSA, E.O. Seletividade de chlorfenapyr e fenbutatin-oxide sobre duas espécies de ácaros predadores (Acari: Phytoseiidae) em citros. Revista Brasileira de Fruticultura, v.23, p.584-588, 2001.

SATO, M.E.; CERÁVOLO, L.C.; CEZÁRIO, A.C.; RAGA, A.; MONTES, S.M.N.M. Toxicidade residual de acaricidas a Euseius citrifolius Denmark \& Muma, 1970 (Acari: Phytoseiidae) em citros. Revista de Agricultura, v.69, p.257-268, 1994.

SATO, M.E.; RAGA, A.; CERÁVOLO, L.C.; ROSSI, A.C.; SOUZA FILHO, M.F. de. Toxicidade residual de acaricidas a Iphiseiodes zuluagai Denmark \& Muma, 1972 (Acari: Phytoseiidae). Arquivos do Instituto Biológico, v.63, p.15-19, 1996.
SATO, M.E.; SILVA, M. DA; GONÇALVES, L.R.; SOUZA FILHO, M.F. DE; RAGA, A. Toxicidade diferencial de agroquímicos a Neoseiulus californicus (McGregor) (Acari: Phytoseiidae) e Tetranychus urticae Koch (Acari: Tetranychidae) em morangueiro. Neotropical Entomology, v.31, p.449-456, 2002.

SILVA, M.Z. Potencialidade do Neoseiulus californicus (McGregor) (Acari: Phytoseiidae) na predação de ácaros fitófagos na cultura dos citros no Estado de São Paulo. 2005. 79f. Dissertação (Mestrado em Agronomia) - Faculdade de Ciências Agrárias e Veterinárias, Universidade Estadual Paulista, Jaboticabal.

SILVA, M.Z.; OLIVEIRA, C.A.L. Seletividade de alguns Agrotóxicos em uso na citricultura ao ácaro predador Neoseiulus californicus (McGregor) (Acari: Phytoseiidae). Revista Brasileira de Fruticultura, v.28, p.205-208, 2006.

SILVA, M.Z.; OLIVEIRA, C.A.L. Toxicidade de alguns agrotóxicos recomendados na citricultura sobre Neoseiulus californicus (McGregor) (Acari: Phytoseiidae). Revista Brasileira de Fruticultura, v.29, p.85-90, 2007.

STRICKLER, K.A.; CROFT, B.A. Selection for permethrin resistance in the predatory mite, Amblyseius fallacis Garman (Acarina: Phytoseiidae). Entomologia Experimentalis et Applicata, v.31, p.339-345, 1982.

van de VRIE, M.; McMURTRY, J.A.; HUFFAKER, C.B. Ecology of tetranychid mites and their natural enemies: A review. III. Biology, ecology, and pest status, and host-plant relations of tetranychids. Hilgardia, v.41, p.387-403, 1972.

ZACHARDA, M.; HLÜCHY, M. Long-term residual efficacy of commercial formulations of pesticides to Typhlodromus pyri Scheuten (Acari: Phytoseiidae) inhabiting commercial vineyards. Experimental and Applied Acarology, v.13, p.27-40, 1991. 